\title{
MORTALIDADE MATERNA POR DOENÇAS HIPERTENSIVAS NO PIAUÍ
}

\section{Maternal mortality from hypertensive diseases in Piauí}

Mortalidad materna por enfermedades hipertensivas em Piauí

Elton Filipe Pinheiro de Oliveira• Mestrando em Saúde e Comunidade pelo Programa de Pós-Graduação em Saúde e Comunidade da Universidade Federal do Piauí (PPGSC/UFPI) • E-mail: helton-oliver@hotmail.com

Andressa Lima Ramos• Mestre em Saúde e Comunidade pelo Programa de PósGraduação em Saúde e Comunidade da Universidade Federal do Piauí (PPGSC/UFPI)・E-mail: andressalimaramos@gmail.com

Malvina Thais Pacheco Rodrigues • Coordenação do Programa de Pós-Graduação em Saúde e Comunidade da Universidade Federal do Piaú (PPGSC/UFPI) E-mail: malvinat@gmail.com

Autor responsável pela correspondência:

Elton Filipe Pinheiro de Oliveira • E-mail: helton-oliver@hotmail.com 


\section{ciência
pural}

\section{RESUMO}

Introdução: A mortalidade materna é considerada um problema de saúde pública, tendo a hipertensão arterial como uma das principais causas obstétricas diretas. Objetivo: Descrever o perfil epidemiológico da mortalidade materna por doenças hipertensivas no Piauí, nos anos de 2007 a 2016. Métodos: Trata-se de um estudo descritivo, com abordagem quantitativa, que utilizou informações de óbitos maternos relacionados à hipertensão arterial da base de dados do Sistema de Informação da Mortalidade e de nascidos vivos do Sistema de Informação sobre Nascidos Vivos dos anos de 2007 a 2016, no Piauí, disponíveis no DATASUS. Resultados: Dos 441 óbitos maternos, 103 (23,3\%) foram relacionados à doença hipertensiva. A maioria dos óbitos ocorreu no interior do estado (86,4\%), no ano de 2008 (20,4\%), no hospital (88,3\%), no puerpério $(65,0 \%)$, sendo a eclâmpsia a principal causa de morte $(67,0 \%)$. As mulheres que mais morreram foram as pardas $(67,9 \%)$, casadas $(29,1 \%)$ e solteiras $(29,1 \%)$, na faixa etária de 20 a 29 anos $(40,8 \%)$ e com oito a onze anos de estudo (20,4\%). A Razão de Mortalidade Materna para o período foi de 20,8 mortes/100 mil nascidos vivos. Conclusões: As doenças hipertensivas constituem-se como uma das principais causas de óbito materno no Piauí, cuja implementação das políticas de enfrentamento vigentes não tem sido suficiente para determinar um padrão decrescente na ocorrência dos óbitos por essas causas.

Palavras-chave: Mortalidade materna. Hipertensão. Saúde da mulher.

\section{ABSTRACT}

Introduction: Maternal mortality is considered a public health problem, having hypertension as one of the main direct obstetric causes. Objective: To describe the epidemiological profile of maternal mortality from hypertensive disease in Piauí, from 2007 to 2016. Methods: This is a descriptive study, with a quantitative approach, that collected data of maternal deaths related to hypertension from the Mortality Information System (SIM) database and live births from the National Live Birth Information System (SINASC) from 2007 to 2016, in Piauí, available on DATASUS. Results: Out of 441 maternal deaths, 103 deaths $(23.3 \%)$ were related to hypertensive disease. Most of the deaths $(86,4 \%)$ occurred in towns and cities not including the capital (Teresina), in 2008 (20.4\%), in hospital $(88.3 \%)$, in puerperium $(65.0 \%)$, eclampsia being the main cause of death $(67.0 \%)$. Women who died the most were the brown ones $(67.9 \%)$, married $29.1 \%$ ) and single women $(29.1 \%)$, aged $20-29$ years old $(40.8 \%)$ and $8-11$ years study $(20.4 \%)$. The Maternal Mortality Ratio for that period was 20.8 deaths 100,000 live births. Conclusions: Hypertensive diseases are one of the main ses of maternal death in Piauí, whose implementation of the current 
confronting policies have not been enough to determine a descending pattern of the number of deaths resulting from those causes.

Keywords: Maternal mortality. Hypertension. Women's health.

\section{RESUMEN}

Introducción: la mortalidad materna se considera un problema de salud pública, con la hipertensión arterial como una de las principales causas obstétricas directas. Objetivo: Describir el perfil epidemiológico de la mortalidad materna por enfermedad hipertensiva en Piauí, de 2007 a 2016. Métodos: Este es un estudio descriptivo, con un enfoque cuantitativo, que utilizó información sobre muertes maternas relacionadas con la hipertensión en la base de datos del Sistema de información sobre mortalidad y nacimientos vivos del Sistema de información sobre nacimientos vivos desde 2007 hasta 2016, en Piauí, disponible en DATASUS. Resultados: De las 441 muertes maternas, 103 (23.3\%) estaban relacionadas con la enfermedad hipertensiva. La mayoría de las muertes ocurrieron en el interior del estado (86.4\%), en 2008 (20.4\%), en el hospital (88.3\%), en el puerperio (65.0\%), siendo la eclampsia la principal. causa de muerte $(67.0 \%)$. Las mujeres que más murieron fueron mulatas $(67.9 \%)$, casadas (29.1\%) y solteras (29.1\%), de 20 a 29 años $(40.8 \%$ ) y de 8 a 11 años. años de estudio (20.4\%). La tasa de mortalidad materna para el período fue de 20.8 muertes / 100 mil nacimientos vivos. Conclusiones: las enfermedades hipertensivas son una de las principales causas de muerte materna en Piauí, cuya implementación de las políticas de afrontamiento actuales no ha sido suficiente para determinar un patrón decreciente en la ocurrencia de muertes por estas causas.

Palabras-clave: Mortalidad materna. Hipertensión. La salud de la mujer. 


\section{Introdução}

Define-se morte materna como a morte de mulheres durante a gestação ou dentro de um período de 42 dias após o término da gravidez devido a qualquer causa relacionada ou agravada pela gravidez ou por medidas tomadas em relação a ela, porém, não devido a causas acidentais ou incidentais ${ }^{1}$.

Os óbitos maternos podem ser divididos em causas obstétricas diretas, indiretas e não especificadas. As causas obstétricas diretas estão relacionadas com complicações ligadas ao ciclo gravídico-puerperal, associadas ou não à má qualidade da assistência prestada à saúde da mulher na gestação, parto ou puerpério $^{2}$. As causas obstétricas indiretas da morte materna decorrem de condições pré-existentes, anteriores à gestação, que se acentuam com a gestação. Ambos os tipos de classificação refletem a má assistência à gestante, acarretando um desfecho desfavorável, cuja evitabilidade está associada ao desenvolvimento de ações assistenciais de qualidade, tratamento precoce e eficaz $^{3}$.

Apesar dos óbitos maternos serem evitáveis em quase 95\% dos casos, estima-se que, no mundo, a cada minuto, uma mulher morre por complicações no parto e com maior proporção de óbitos em países em desenvolvimento, configurando-se como um problema de saúde $e^{4,5}$.

Em 2015, a taxa de mortalidade materna nos países em desenvolvimento foi de 239 mortes/100 mil Nascidos Vivos (NV), enquanto que nos países desenvolvidos atingiu 12 mortes/100 mil NV. Neste ano, em todo o mundo, cerca de 303 mil mulheres morreram durante e após a gravidez e o parto. Existem grandes disparidades entre os países e dentro dos países, entre mulheres com baixas e altas rendas e entre a população rural e a população urbana $^{5}$.

Ainda em 2015, o Brasil registrou 1.738 casos de morte materna, dos quais foram registrados na região Nordeste, segunda região brasileira onde mais 
registraram óbitos maternos. Neste cenário, o Piauí ocupa a $15^{\mathrm{a}}$ posição nacional e a quinta posição regional, sendo as doenças hipertensivas responsáveis por quase $22 \%$ do total de mortes materna ${ }^{6}$.

Nesta perspectiva, este estudo tem como objetivo descrever o perfil epidemiológico da mortalidade materna por doenças hipertensivas no Piauí, nos anos de 2007 a 2016.

\section{Método}

A pesquisa caracteriza-se por ser do tipo quantitativa e descritiva, que utilizou dados secundários do Sistema de Informação sobre Mortalidade (SIM). Para o cálculo da Razão de Mortalidade Materna (RMM) foram considerados os dados de NV no Piauí, nos anos de 2007 a 2016, disponíveis do Sistema de Informação sobre Nascidos Vivos (SINASC).

A amostra deste estudo foi composta pelos óbitos maternos relacionados às doenças hipertensivas (CID-10 (O13, O14, O15 e O16)), no período de 2007 a 2016, ocorridos no estado do Piauí. A coleta dos dados ocorreu no mês de janeiro/2019.

As variáveis estudadas foram: ano de ocorrência, faixa etária, escolaridade (anos de estudo), raça/cor, estado civil, local de ocorrência, causas (CID-10), período de ocorrência (ciclo gravídico-puerperal) e RMM.

Os dados foram organizados em planilha do Excel® 2010 e foram analisados por meio de estatística descritiva, com o auxílio do programa StatisticalPackage for Social Sciences-SPSS 20.0. Foram calculadas as medidas centrais para as variáveis contínuas e medidas de frequência para as variáveis categóricas.

A submissão junto ao Comitê de Ética em Pesquisa não foi necessária, oor se tratar da utilização de base de dados secundários, onde não há dentificação de indivíduos. 


\section{ciência
pural}

\section{Resultados}

Entre 2007 a 2016, dos 441 óbitos maternos ocorridos no Piauí, 103 foram relacionados às doenças hipertensivas. A maior ocorrência foi evidenciada em 2008 (20,4\%). De 2011 até 2013 observou-se redução no número de casos, enquanto que nos anos de 2014 e 2015 ocorreu aumento no número de óbitos, quando comparados ao ano de 2013. Em 2016, houve redução da ocorrência de casos quando comparado aos anos anteriores (2013, 2014 e 2015) (Figura 1).

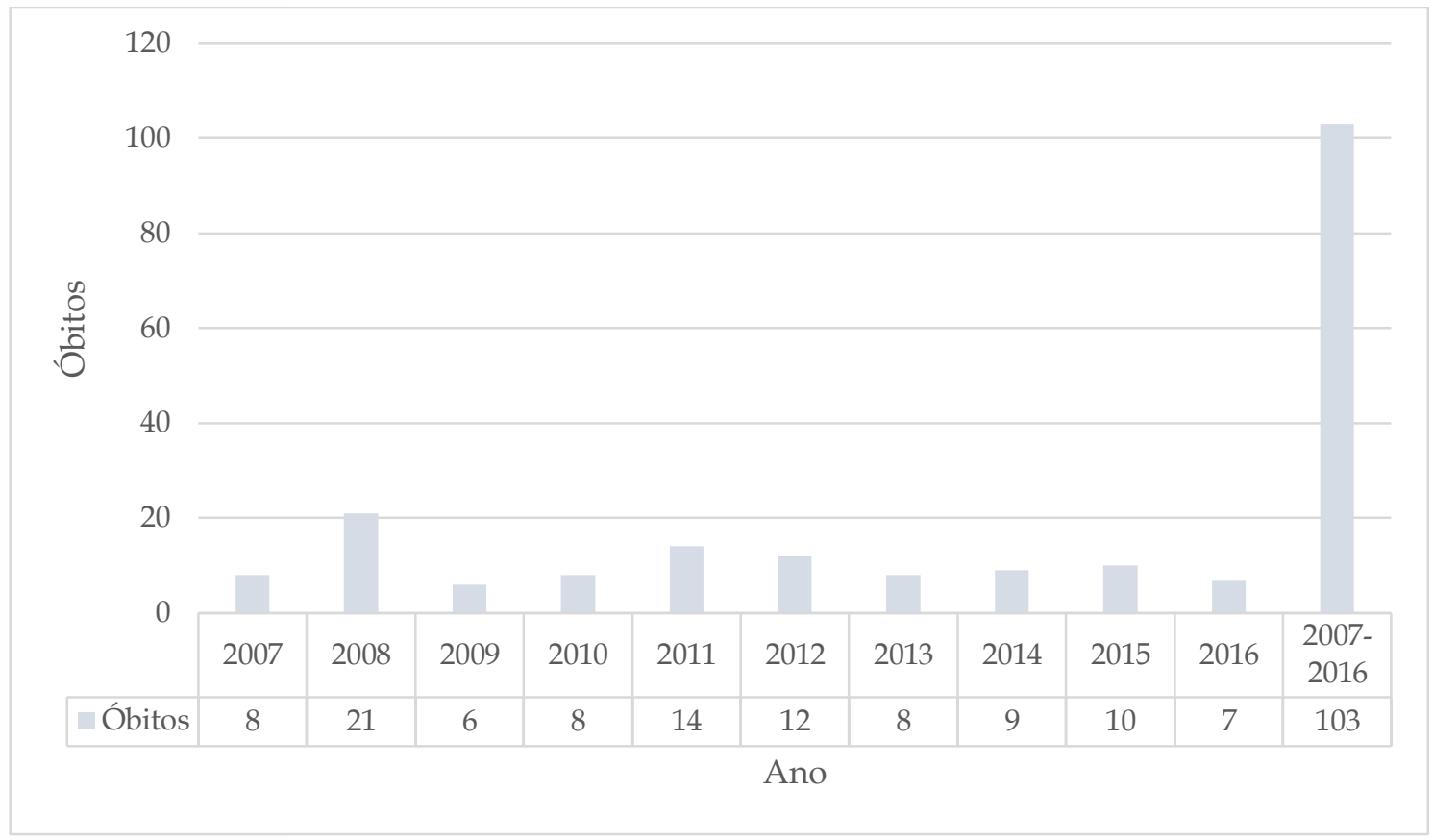

Figura 1. Distribuição dos óbitos maternos por doenças hipertensivas, Piauí, Brasil, 2007 a 2016. Teresina-PI, 2019.

Fonte: Sistema de Informação sobre Mortalidade-SIM (2019)

Os óbitos foram mais frequentes em mulheres com 20 a 29 anos, com oito a 11 anos de estudo e pardas. Não houve diferença percentual entre os grupos casadas e solteiras, sendo esses os mais prevalentes na variável estado civil. local mais informado para a ocorrência destes óbitos foi o hospital $(88,4 \%)$ e 


\section{ciência
pural}

a maior parte dos óbitos ocorreram no interior do estado $(86,4 \%)$. Teresina (capital) registrou a maior frequência de óbitos (13,6\%), seguida de Parnaíba, segunda cidade mais populosa do Piauí, com seis casos (5,8\%). (Tabela 1)

Tabela 1. Distribuição dos aspectos sociodemográficos dos óbitos maternos por doenças hipertensivas, Piauí, Brasil, 2007 a 2016. Teresina-PI, 2019.

\begin{tabular}{lcc} 
Variáveis & n & \% \\
\hline Faixa etária & 18 & 17,7 \\
10 a 19 & 42 & 40,7 \\
20 a 29 & 38 & 36,8 \\
30 a 39 & 5 & 4,8 \\
40 a 49 & & \\
& & \\
Escolaridade (anos de estudo) & 7 & 6,8 \\
Nenhuma (0) & 11 & 10,7 \\
1 a 3 & 19 & 18,4 \\
4 a 7 & 21 & 20,5 \\
8 a 11 & 9 & 8,7 \\
Mais de 12 & & \\
Raça/cor* & & 14,6 \\
Branca & 15 & 8,7 \\
Preta & 9 & 0,9 \\
Amarela & 1 & 68,0 \\
Parda & 70 &
\end{tabular}

\section{Estado civil*}

Casada

Solteira

Viúva

Separada

Outros

Local de ocorrência

Hospital

Domicílio 


\begin{tabular}{lcc}
\hline Local/Município & 14 & \\
Capital & 13,6 \\
Interior & 89 & 86,4 \\
& & \\
Teresina & 14 & 13,6 \\
Parnaíba & 6 & 5,8 \\
Oeiras & 5 & 4,8 \\
Floriano & 4 & 3,9 \\
Picos & 3 & 2,9 \\
Luzilândia & 3 & 2,9 \\
Barras & 3 & 2,9 \\
Simplício Mendes & 2 & 1,9 \\
Piripiri & 2 & 1,9 \\
Pedro II & 2 & 1,9 \\
\hline
\end{tabular}

Fonte: Sistema de Informação sobre Mortalidade-SIM (2019)

* Excluídas as informações ignoradas.

O período de maior ocorrência dos óbitos, segundo o ciclo gravídicopuerperal, foi puerpério $(65,2 \%)$ e a principal causa de morte foi a eclampsia, responsável por $67 \%$ dos óbitos maternos (Tabela 2).

Tabela 2. Distribuição do período de ocorrência segundo o ciclo gravídicopuerperal e causas pela CID-10 dos óbitos maternos, Piauí, Brasil, 2007 a 2016. Teresina-PI, 2019.

\begin{tabular}{|c|c|c|}
\hline Variáveis & $\mathbf{n}$ & $\%$ \\
\hline \multicolumn{3}{|l|}{ Período de ocorrência dos óbitos } \\
\hline Gravidez, parto ou aborto & 30 & 29,1 \\
\hline Puerpério, até 42 dias após o parto & 67 & 65,2 \\
\hline Puerpério, de 43 dias a menos de 1 ano (Tardio) & 1 & 0,9 \\
\hline Período não informado/Ignorado & 5 & 4,8 \\
\hline \multicolumn{3}{|l|}{ Causas } \\
\hline $\begin{array}{l}\text { Hipertensão gestacional sem proteinúria significativa } \\
\text { (O13) }\end{array}$ & $\begin{array}{c}2 \\
31\end{array}$ & $\begin{array}{r}1,9 \\
30,2\end{array}$ \\
\hline Hipertensão gestacional com proteinúria significativa & 69 & 67,0 \\
\hline 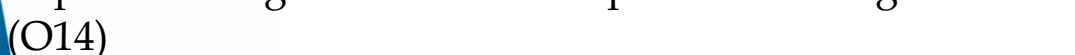 & 1 & 0.9 \\
\hline Eclâmpsia (O15) & & \\
\hline Hipertensão materna não específica (O16) & & \\
\hline
\end{tabular}

Fonte: Sistema de Informação sobre Mortalidade-SIM (2019) 
A razão de Mortalidade Materna (RMM) variou durante o período estudado, sendo maior no ano de 2008, correspondendo a 39,9 mortes/100 mil NV e menor em 2009 com 11,8 mortes/100 mil NV (Tabela 3).

Tabela 3. Razão de Mortalidade Materna (RMM), segundo ano de ocorrência dos óbitos maternos por doenças hipertensivas, Piauí, Brasil, 2007 a 2016. Teresina-PI, 2019.

\begin{tabular}{cccc}
\hline Ano & NV & Óbitos & RMM \\
\hline 2007 & 53.214 & 8 & 15,0 \\
2008 & 52.664 & 21 & 39,9 \\
2009 & 50.996 & 6 & 11,8 \\
2010 & 49.424 & 8 & 16,2 \\
2011 & 50.144 & 14 & 27,9 \\
2012 & 47.962 & 12 & 25,0 \\
2013 & 46.419 & 8 & 17,2 \\
2014 & 47.941 & 9 & 18,8 \\
2015 & 49.253 & 10 & 20,3 \\
2016 & 46.986 & 7 & 14,9 \\
Total (2007-2016) & 495003 & 103 & 20,8 \\
\hline
\end{tabular}

Fonte: Sistema de Informação sobre Mortalidade-SIM (2019); Sistema de Informação sobre Nascidos Vivos-SINASC (2019)

\section{Discussão}

As mortes maternas relacionadas às doenças hipertensivas ocorreram, principalmente, em decorrência de complicações da eclampsia, no período do puerpério. A maioria das mulheres eram jovens, no período reprodutivo, pardas, com oito a 11 anos de estudo e provenientes do interior do estado. A RMM por doenças hipertensivas em todo o período estudado foi de 20,8 mortes/100 mil NV. 
O total de óbitos maternos por doenças hipertensivas evidenciados nesse estudo foi $30,4 \%$ maior que aquele verificado em estudo retrospectivo ${ }^{8}$, realizado no estado de Santa Catarina, que utilizou dados secundários do SIM, no período de 10 anos.

A análise da série histórica dos óbitos maternos apontou um padrão oscilatório, com ascensão e declínio no número de óbitos entre os anos de 2007 e 2016, com destaque para o crescimento expressivo evidenciado em 2008. Esse padrão de oscilação, apresentado ao longo dos anos, é preocupante, visto que foram criadas e implementadas algumas políticas públicas, no âmbito do Sistema Único de Saúde, como a Rede Cegonha, implantada pela portaria $\mathrm{n}^{\mathrm{o}}$ 1459 de 24 de junho de $2011^{1^{0}}$, que preconiza a organização da rede de assistência à saúde materno-infantil, na perspectiva de melhoria na qualidade das ações oferecidas com conseqüente redução da mortalidade materna.

Nos anos de 2013 a 2015, a mortalidade materna apresentou tendência de decréscimo no Nordeste $(-2,9 \%)$ e no Brasil $(-2,7 \%)^{6}$. Contrariando a tendência de redução, o Piauí apresentou no mesmo período, aumento de 20\% no total de óbitos maternos por doenças hipertensivas. Em 2016 observou-se uma redução nos casos ocorridos no Piauí, seguindo a tendência regional e nacional ${ }^{6}$. Este fato pode estar associado à redução no número de NV registrados no Piauí no referido ano, além da intensificação das ações para a redução da mortalidade materna nacional ${ }^{5}$.

A ocorrência desses óbitos foi observada, majoritariamente, em mulheres com idade de 20 a 29 anos, ou seja, na idade reprodutiva ${ }^{16}$. Este achado está em consonância com outros estudos retrospectivos recentes ${ }^{7}, 12,13$ envolvendo a mortalidade materna e que apontam que as mulheres morrem com maior freqüência na idade considerada fértil. É nessa faixa etária que ocorre maior uúmero de gestações $^{20}$, refletindo a necessidade de melhoria nas ações de lanejamento familiar, em toda sua completude, especialmente no pré-natal. 
A baixa escolaridade é considerada fator de risco e contribui para a ocorrência dos óbitos maternos por doenças hipertensivas. Estudo aponta relação direta entre poucos anos de estudo e mortalidade materna ${ }^{13}$. O menor tempo de estudo está associado ao baixo acesso à informação e menos conhecimentos sobre hábitos de saúde, principalmente no que diz respeito ao pré-natal a à saúde reprodutiva. A baixa escolaridade gera repercussões não apenas relacionadas ao trabalho e renda, mas também na saúde da população, em especial, de mulheres na idade reprodutiva ${ }^{14}$.

No Brasil, cerca de $41 \%$ das pessoas autodeclaram-se pardas ${ }^{18}$, sendo essa a raça/cor mais prevalente neste estudo. Resultados semelhantes foram achados em outros estudos que apontam uma maior ocorrência de óbitos maternos entre as mulheres não brancas ${ }^{7,14}$. Os resultados encontrados neste estudo reforçam a hipótese de que as mulheres não brancas constituem um grupo vulnerável para a morte materna, por apresentarem, geralmente, baixa condição socioeconômica ${ }^{3}$. Contudo, essa particularidade pode ser associada à região em que tais óbitos ocorrem, visto que, estudo realizado no Sul do país ${ }^{17}$ apontou uma maior ocorrência dos óbitos maternos entre as mulheres consideradas brancas.

Entre as mulheres, sabe-se que nas relações conjugais, o aporte afetivo, emocional, social e financeiro à mulher e o estímulo ao autocuidado à mãe pela presença ou não do pai do bebê e da família são fatores de proteção relevantes para a prevenção da morte materna, sendo a ausência do companheiro, evidenciada como um fator de risco para o óbito materno ${ }^{3}$. Apesar disso, a maior prevalência dos óbitos maternos foram verificadas entre as mulheres casadas ou solteiras, em consonância com outros estudos 11,13,15.

Os óbitos maternos têm como principal local de ocorrência o ambiente ospitalar, sendo esse o principal destino das gestantes para realização do parto ou para serem atendidas em decorrência de complicações da hipertensão erial descompensada ${ }^{3}$. O hospital é, frequentemente, apontado pela 
qualidade da assistência prestada, muitas vezes por não apresentar suporte adequado à gestante. Outra situação que pode estar associada à letalidade dos casos é o grau da gravidade e condições em que essas gestantes são encaminhadas ao hospital, o que pode ser resultante de uma assistência prénatal insuficiente e incapaz de identificar os sinais precoce de complicações da gestação na atenção primária' ${ }^{12,20}$.

A grande maioria dos óbitos maternos por doenças hipertensivas foram registrados em cidades do interior do Piauí. Geralmente, esses municípios apresentam fragilidades que determinam a magnitude da mortalidade materna, principalmente, na atenção primária à saúde, pela incapacidade na identificação e monitoramento das gestantes de alto risco, principalmente as hipertensas, gerando a necessidade de atendimento hospitalar para tratar de complicações decorrentes da hipertensão na gestação ${ }^{20}$. Esse cenário não é restrito ao Piauí. Nos demais estados do país também se observa que as mortes maternas ocorrem, na maioria dos casos, em cidades do interior ${ }^{6}$.

A maior incidência dos óbitos maternos, seja na capital, ou nas cidades do interior, ocorre no período do puerpério (até 42 dias após o parto) também foi encontrada em estudo epidemiológico ${ }^{3}$, realizado no Ceará, abordando as causas hipertensivas e hemorrágicas da morte materna. No puerpério ocorrem inúmeras alterações fisiológicas que podem acentuar as complicações decorrentes da hipertensão na gestação ${ }^{20}$, sendo de extrema importância a manutenção dos cuidados dispensados à puérpera, tais como vigilância da pressão arterial, sinais de infecção, choque hipovolêmico e hemorragias ${ }^{19}$.

Dentre as causas de morte materna, as obstetras diretas são responsáveis por cerca de $63 \%$ do total de óbitos maternos ${ }^{17}$, sendo evitáveis na grande maioria dos casos. A hipertensão arterial é descrita como a principal causa obstétrica direta, responsável por até $28,1 \%$ do total de óbitos maternos, em estudo realizado em capital nordestina ${ }^{20}$. No contexto das causas hipertensivas, clâmpsia foi apontada como a principal causa de morte de gestantes em 
diversos estudos realizados em diferentes estados do país ${ }^{7,8,13}$, pelas repercussões ocasionadas no organismo materno decorrente da hipertensão, edema e convulsões.

O monitoramento adequado das gestantes com essa condição impactaria de forma significativa na redução da RMM nacional, que é de 68 mortes/100 mil NV. Neste estudo, a RMM por doenças hipertensivas apresentou tendência variada e atingiu mais de 2/3 da RMM (30 mortes/100 mil NV) firmada como meta para ODS para o período de 2015-2030 e que inclui todas as causas de morte materna. Esse valor demonstra o alto impacto das causas hipertensivas na mortalidade materna. Comportamento semelhante foi observado em estudo sobre a mortalidade materna por hipertensão, abordando série histórica diferente ${ }^{8}$.

Considerando que a hipertensão é apontada como uma das principais causas de morte materna ${ }^{18}$, o diagnóstico precoce e adequado acompanhamento das mulheres em idade reprodutiva e gestantes com essa condição minimizariam a ocorrência de complicações decorrentes da hipertensão arterial, e consequentemente, culminariam na redução da mortalidade materna por essas causas.

As limitações do estudo estão relacionadas à utilização de dados secundários pela qualidade das informações prestadas durante $\mathrm{o}$ preenchimento de ficha utilizadas na alimentação dos sistemas de informações utilizados.

\section{Conclusões}

As doenças hipertensivas constituem-se como uma das principais causas ébito materno no Piauí, cuja implementação das políticas de enfrentamento igentes não tem sido suficiente para determinar um padrão decrescente na corrência dos óbitos por essas causas. 
Tornam-se necessárias ações de monitoramento mais eficazes a fim de garantir uma assistência integral à mulher em todo o ciclo gravídico puerperal, visto que as doenças hipertensivas são consideradas causas sensíveis à atenção primária.

Estudos epidemiológicos periódicos sobre o assunto são necessários a fim de subsidiar a tomada de decisão e a implementação de forma mais efetiva das políticas públicas de atenção à saúde da mulher.

\section{Referências}

1. Ministério da Saúde (BR). Secretaria de Atenção à Saúde. Departamento de Ações Programáticas Estratégicas. Gestação de Alto Risco: Manual Técnico [Internet]. 5th ed. Brasília. Ministério da Saúde. 2012. [citado 03 jan 2019]. Disponível em:

http:/ /bvsms.saude.gov.br/bvs/publicacoes/manual_tecnico_gestacao_alto_ risco.pdf

2. Tognini S. Tendência da mortalidade materna na região do Grande $A B C$ Paulista de 1997 a 2011 [dissertação]. São Paulo (SP): Universidade de São Paulo; 2014.

3. Sousa DMN.; Mendes ICM.; Oliveira ET.; Chagas ACMA.; Catunda HLO.; Oriá MOB. Mortalidade materna por causas hipertensivas e hemorrágicas: análise epidemiológica de uma década. Rev enferm UERJ. Rio de Janeiro. 2014. jul/ago; 22(4):500-6.

4. Faria DR.; Sousa RC.; Costa TJNM.; Leite ICG. Mortalidade materna em cidade-polo de assistência na região Sudeste: tendência temporal e determinantes sociais. Ver Méd Minas Gerais [Internet]. 2012. [citado em 20 de mar 2019];22(1):1-8. Disponível em:

http:/ / http://www.rmmg.org/artigo/detalhes/121

World Health Organization. WHO. Maternal mortality [Internet]. 2016.

Datasus. Sistema de informação de Informação sobre mortalidade-SIM. tado em 20 mar 2019]. Disponível em:

ttp:/ / www2.datasus.gov.br/DATASUS/index.php?area=0205\&id=6937 


\section{ciênncia
purál}

7. Gomes JO.; Vieira MCA.; Mistura C.; Andrade GG.; Barbosa KMG.;

Carvalho e Lira MOS, et al. Perfil sociodemográfico e clínico de mortalidade materna. revenferm UFPE online. Recife, dez. 2018 [citado 12 mar 2019], 12(12):3165-71. Disponível em: https://doi.org/10.5205/1981-8963v12i12a237316p3165-3171-2018.

8. Saviato B.; Knobel R.; Moraes CA.;Tonon D. Morte materna por hipertensão no Estado de Santa Catarina. Arquivos Catarinenses de Medicina. 2008, 37(4): 16-19

9. Departamento de Informática do Sistema Único de Saúde (DATASUS). Sistema de informação sobre Nascidos Vivos-SINASC. [citado em 12 jan 2019]. Disponível em:

http://tabnet.datasus.gov.br/cgi/deftohtm.exe?sinasc/cnv/nvPI.def

10. Morse ML.; Fonseca SC.; Barbosa MD.; Calil MD.;Eyer FPC. Mortalidade Materna no Brasil: o que mostra a produção científica nos últimos 30 anos? Cad Saúde Pública [Internet]. 2008. [citado em 15 fev 2019];27(4):623-38. Disponível em: http://https://www.scielo.br/pdf/csp/v27n4/02.pdf.

11. Martins ACS.; Silva LS. Perfil epidemiológico de mortalidade materna. RevBrasEnferm [Internet]. 2018 [citado em 15 fev 2019], 71(supl1):725-31. Disponível em: http://dx.doi.org/10.1590/0034-7167-2017-0624.

12. Gois EC.; Jesus CS.; Conceição GC.; Santos JB.; Reis RB. Mortalidade materna na Bahia no período de 2012 a 2016. REAS [Internet].2019 [citado 9 ago 2019];(18): 1-8; e335. Disponível em:

https://acervomais.com.br/index.php/saude/article/view/335

13. Medeiros LT.; Sousa AM.; Arinana LO.; Inácio AS.; Prata MLC.;

Vasconcelos MNG. Mortalidade materna no estado do Amazonas: estudo epidemiológico. Rev baiana enferm. 2018;(32): 1-11; e26623.

14. Marinho ACN.; Paes NA. Mortalidade materna no estado da Paraíba: associação entre variáveis. Rev Esc Enferm USP. 2010; 44(3): 732-8.

15. Júnior GF.; Matias JG.; Soares MSAC. Profile partner epidemilogical of maternal deaths ocurred in the state of Piauí. Rev Interd Ciên Saúde [Internet]. 2015 Aug/Oct. [citado 03 mar 2019];2(3):47-55. Disponível em: http://https://revistas.ufpi.br/index.php/rics/article/download/2128/2355

Vega CEP.; Soares VMN.;Nasr AMLF. Mortalidade materna tardia: mparação de dois comitês de mortalidade materna no Brasil. Cad Saúde 
Pública [online]. 2017 [citado 03 abr 2019];33(3): 1-13; e00197315. DOI: 10.1590/0102-311X00197315.

17. Sombrio SN.; Simões PW.; Medeiros LR.; Silva FR.; Silva BR.; Rosa MI.; Farias BF. Razão de mortalidade materna na região sul do Brasil no período de 1996 a 2005. ACM Arq Catarin Med. 2011; 40(3): 56-62.

18. Instituto Brasileiro de Geografia e Estatística. Cor ou raça (Brasil, 2015). Rio de Janeiro; 2015.

19. Costa AAR.; Ribas MSSS.; Amorim MMR.; Santos LC. Mortalidade Materna na Cidade do Recife. RBGO2002, 24(7): 455-462.

20. Herculano MMS.; Veloso LS.; Teles LMR.; Oriá MOB.; Almeida PC.;

Damasceno AKC. Óbitos maternos em uma maternidade pública de Fortaleza: um estudo epidemiológico. Rev Esc Enferm USP. 2012, 46 (2): 395-301 